\title{
Synthesis, Crystal structure, and Properties of the Alluaudite-Type Vanadates $\mathrm{Ag}_{2-\mathrm{x}} \mathrm{Na}_{\mathrm{x}} \mathrm{Mn}_{2} \mathrm{Fe}\left(\mathrm{VO}_{4}\right)_{3}$
}

Hamdi Ben Yahia, ${ }^{\mathrm{a}, \mathrm{b}, *}$ Masahiro Shikano, ${ }^{\mathrm{b}, *}$ Mitsuharu Tabuchi, ${ }^{\mathrm{b}}$ and Ilias Belharouak ${ }^{\mathrm{a}}$

${ }^{a}$ Qatar Environment and Energy Research Institute, Hamad Bin Khalifa University, Qatar

Foundation, P.O. Box 5825 Doha, Qatar.

${ }^{\mathrm{b}}$ Research Institute of Electrochemical Energy, National Institute of Advanced Industrial Science and Technology (AIST), 1-8-31 Midorigaoka, Ikeda, Osaka 563-8577, Japan.

Corresponding authors: *Hamdi Ben Yahia, E-mail: Hyahia@qf.org.qa, Fax: +974-4454 0547; Tel: +974-4454-7762 and *Masahiro Shikano, E-mail: shikano.masahiro@aist.go.jp, Fax: +81-72-7519609; Tel: +81-72-751-8460. 


\section{Supplementary information}

Table S1. Alluaudite compounds containing silver cations in the ICSD.

\begin{tabular}{lllllll}
\hline $\mathbf{C o m p o u n d s}$ & $\boldsymbol{a}(\AA)$ & $\boldsymbol{b}(\AA)$ & $\boldsymbol{c}(\AA)$ & $\boldsymbol{\beta}\left(^{\circ}\right)$ & $\boldsymbol{V}\left(\AA^{3}\right)$ & Ref. \\
\hline $\mathrm{Ag}_{3} \mathrm{Tl}_{2}\left(\mathrm{PO}_{4}\right)_{3}$ & $13.138(3)$ & $13.111(3)$ & $6.7254(13)$ & $114.62(3)$ & 1053.15 & 8 \\
$\mathrm{Ag}_{3} \mathrm{In}_{2}\left(\mathrm{PO}_{4}\right)_{3}$ & $12.6305(1)$ & $12.8549(1)$ & $6.5989(1)$ & $113.842(1)$ & 979.99 & 9 \\
$\mathrm{Ag}_{2} \mathrm{FeMn}_{2}\left(\mathrm{PO}_{4}\right)_{3}$ & $12.1466(3)$ & $12.7328(4)$ & $6.4999(5)$ & $114.5323(3)$ & 914.53 & 10 \\
$\mathrm{AgNaFeMn}_{2}\left(\mathrm{PO}_{4}\right)_{3}$ & $12.085(1)$ & $12.684(0)$ & $6.498(4)$ & $114.535(3)$ & 906.12 & 11 \\
$\mathrm{Ag}_{0.98} \mathrm{Ca}_{0.786} \mathrm{Cd}_{1.114} \mathrm{Mg}_{2.14}\left(\mathrm{PO}_{4}\right)_{3}$ & $12.106(3)$ & $12.677(2)$ & $6.525(1)$ & $114.67(2)$ & 909.98 & 12 \\
$\mathrm{Ag}_{0.86} \mathrm{Cd}_{1.984} \mathrm{Mg}_{2.34}\left(\mathrm{PO}_{4}\right)_{3}$ & $12.089(3)$ & $12.653(2)$ & $6.530(1)$ & $114.71(2)$ & 907.38 & 12 \\
$\mathrm{AgMn}_{3}\left(\mathrm{PO}_{4}\right)\left(\mathrm{HPO}_{4}\right)_{2}$ & $12.263(1)$ & $12.446(2)$ & $6.649(1)$ & $114.708(8)$ & 921.90 & 13 \\
$\mathrm{AgCo}_{3}\left(\mathrm{PO}_{4}\right)\left(\mathrm{HPO}_{4}\right)_{2}$ & $12.035(2)$ & $12.235(2)$ & $6.541(2)$ & $114.14(2)$ & 878.92 & 14 \\
$\mathrm{AgNi}_{3}\left(\mathrm{PO}_{4}\right)\left(\mathrm{HPO}_{4}\right)_{2}$ & $11.865(4)$ & $12.117(3)$ & $6.467(2)$ & $113.82(3)$ & 850.55 & 15 \\
$\mathrm{AgMg}_{3}\left(\mathrm{PO}_{4}\right)\left(\mathrm{HPO}_{4}\right)_{2}$ & $11.9126(5)$ & $12.1197(6)$ & $6.4780(3)$ & $113.812(2)$ & 855.66 & 16 \\
\hline $\mathrm{AgMn}_{3}\left(\mathrm{AsO}_{4}\right)\left(\mathrm{HAsO}_{4}\right)_{2}$ & $12.3966(11)$ & $12.7069(15)$ & $6.8904(6)$ & $113.574(10)$ & 994.81 & 17 \\
$\mathrm{AgCo}_{3}\left(\mathrm{AsO}_{4}\right)\left(\mathrm{HAsO}_{4}\right)_{2}$ & $12.596(15)$ & $12.438(7)$ & $6.782(3)$ & $113.16(3)$ & 943.01 & 18 \\
$\mathrm{AgZn}_{3}\left(\mathrm{AsO}_{4}\right)\left(\mathrm{HAsO}_{4}\right)_{2}$ & $12.169(2)$ & $12.495(3)$ & $6.755(1)$ & $112.77(1)$ & 947.06 & 18 \\
$\mathrm{Ag}_{1.49} \mathrm{Mn}_{3}\left(\mathrm{AsO}_{4}\right)_{3}$ & $12.262(2)$ & $12.934(3)$ & $6.707(1)$ & $113.690(2)$ & 974.07 & 19 \\
\hline $\mathrm{Ag}_{2} \mathrm{Mn}_{3}\left(\mathrm{VO}_{4}\right)_{3}$ & $11.8968(11)$ & $13.2057(13)$ & $6.8132(7)$ & $111.3166(15)$ & $997.16(17)$ & 20 \\
\hline
\end{tabular}


Table S2. Anisotropic displacement parameters $\left(\AA^{2}\right)$ for $\mathrm{Ag}_{2-x} \mathrm{Na}_{x} \mathrm{Mn}_{2} \mathrm{Fe}\left(\mathrm{VO}_{4}\right)_{3}(x=0,5 / 4$, and 2$)$. The anisotropic displacement factor exponent takes the form: $-2 \pi^{2}\left[\left(h a^{*}\right)^{2} U_{11}+\ldots+2 h k a^{*} b^{*} U_{12}\right]$.

\begin{tabular}{|c|c|c|c|c|c|c|}
\hline Atom & $U_{11}$ & $U_{22}$ & $U_{33}$ & $U_{12}$ & $U_{13}$ & $U_{23}$ \\
\hline \multicolumn{7}{|c|}{$\mathrm{Na}_{2} \mathrm{Mn}_{2} \mathrm{Fe}\left(\mathrm{VO}_{4}\right)_{3}$} \\
\hline $\mathrm{Nal}$ & $0.0269(11)$ & $0.0061(9)$ & $0.0067(8)$ & $-0.0029(7)$ & $-0.0027(7)$ & $-0.0015(6)$ \\
\hline $\mathrm{Na} 2$ & $0.0273(14)$ & $0.083(2)$ & $0.0344(13)$ & 0.00000 & $0.0057(11)$ & 0.00000 \\
\hline Mn1 & $0.0124(3)$ & $0.0159(4)$ & $0.0136(3)$ & 0.00000 & $0.0069(3)$ & 0.00000 \\
\hline $\mathrm{Mn} 2 / \mathrm{Fe} 2$ & $0.0138(3)$ & $0.0144(3)$ & $0.0102(2)$ & $-0.00092(18)$ & $0.00597(19)$ & $0.00032(17)$ \\
\hline V1 & $0.0161(4)$ & $0.0120(4)$ & $0.0088(3)$ & 0.00000 & $0.0034(3)$ & 0.00000 \\
\hline $\mathrm{V} 2$ & $0.0117(3)$ & $0.0147(3)$ & $0.0095(3)$ & $-0.0001(2)$ & $0.0043(2)$ & $-0.0012(2)$ \\
\hline O1 & $0.0190(13)$ & $0.0231(14)$ & $0.0210(12)$ & $0.0018(10)$ & $0.0082(10)$ & $-0.004(1)$ \\
\hline $\mathrm{O} 2$ & $0.0207(12)$ & $0.0179(13)$ & $0.0126(11)$ & $0.0051(10)$ & $0.0066(9)$ & $0.0010(9)$ \\
\hline $\mathrm{O} 3$ & $0.0214(12)$ & $0.0220(14)$ & $0.0137(11)$ & $-0.0004(10)$ & $0.0079(10)$ & $-0.0005(9)$ \\
\hline $\mathrm{O} 4$ & $0.0140(12)$ & $0.0189(14)$ & $0.0343(13)$ & $-0.0006(10)$ & $0.0095(10)$ & $-0.0033(11)$ \\
\hline $\mathrm{O} 5$ & $0.0129(11)$ & $0.0230(13)$ & $0.0118(10)$ & $0.0002(10)$ & $0.0053(9)$ & $0.0030(9)$ \\
\hline O6 & $0.0262(13)$ & $0.0193(14)$ & $0.0254(13)$ & $-0.0055(11)$ & $0.0053(11)$ & $0.0037(10)$ \\
\hline \multicolumn{7}{|c|}{$\mathrm{Ag}_{3 / 4} \mathrm{Na}_{5 / 4} \mathrm{Mn}_{2} \mathrm{Fe}\left(\mathrm{VO}_{4}\right)_{3}$} \\
\hline Na1/Ag1 & 0.04366 & 0.01862 & 0.01464 & -0.00887 & 0.00511 & -0.00184 \\
\hline $\mathrm{Na} 2 / \mathrm{Ag} 2$ & 0.01616 & 0.05233 & 0.02270 & 0.00000 & 0.00267 & 0.00000 \\
\hline Mn1 & 0.01234 & 0.01352 & 0.01117 & 0.00000 & 0.00551 & 0.00000 \\
\hline $\mathrm{Mn} 2 / \mathrm{Fe} 2$ & 0.01282 & 0.01182 & 0.00822 & -0.00012 & 0.00414 & 0.00085 \\
\hline V1 & 0.01443 & 0.00975 & 0.00738 & 0.00000 & 0.00245 & 0.00000 \\
\hline $\mathrm{V} 2$ & 0.01198 & 0.01136 & 0.00780 & -0.00014 & 0.00305 & -0.00095 \\
\hline $\mathrm{O} 1$ & 0.01894 & 0.01928 & 0.01988 & 0.00156 & 0.00609 & -0.00383 \\
\hline $\mathrm{O} 2$ & 0.01993 & 0.01691 & 0.00851 & 0.00323 & 0.00197 & 0.00123 \\
\hline $\mathrm{O} 3$ & 0.01957 & 0.02052 & 0.01204 & 0.00083 & 0.00595 & 0.00055 \\
\hline $\mathrm{O} 4$ & 0.01393 & 0.01617 & 0.02964 & -0.00058 & 0.00730 & -0.00168 \\
\hline $\mathrm{O} 5$ & 0.01498 & 0.01973 & 0.00940 & 0.00119 & 0.00446 & 0.00277 \\
\hline O6 & 0.02261 & 0.01622 & 0.02085 & -0.00321 & 0.00329 & 0.00119 \\
\hline \multicolumn{7}{|c|}{$\mathrm{Ag}_{2} \mathrm{Mn}{ }_{2} \mathrm{Fe}\left(\mathrm{VO}_{4}\right)_{3}$} \\
\hline Ag1 & $0.0418(3)$ & $0.0194(2)$ & $0.01865(18)$ & $-0.00958(13)$ & $0.00762(16)$ & $-0.00217(9)$ \\
\hline Ag2 & $0.0183(2)$ & $0.0559(3)$ & $0.0275(2)$ & 0.00000 & $0.00582(16)$ & 0.00000 \\
\hline Mn1 & $0.0100(3)$ & $0.0115(3)$ & $0.0137(2)$ & 0.00000 & $0.00610(19)$ & 0.00000 \\
\hline $\mathrm{Mn} 2 / \mathrm{Fe} 2$ & $0.0103(2)$ & $0.0106(2)$ & $0.01135(19)$ & $-0.00031(13)$ & $0.00512(14)$ & $0.00046(11)$ \\
\hline V1 & $0.0118(3)$ & $0.0085(3)$ & $0.0096(3)$ & 0.00000 & $0.0035(2)$ & 0.00000 \\
\hline $\mathrm{V} 2$ & $0.0085(2)$ & $0.0104(2)$ & $0.0103(2)$ & $-0.00025(14)$ & $0.00340(16)$ & $-0.00039(12)$ \\
\hline $\mathrm{O} 1$ & $0.0171(10)$ & $0.019(1)$ & $0.0257(10)$ & $0.0009(7)$ & $0.0091(9)$ & $-0.0043(6)$ \\
\hline $\mathrm{O} 2$ & $0.0168(9)$ & $0.0155(8)$ & $0.0139(7)$ & $0.0037(7)$ & $0.0042(7)$ & $0.0019(6)$ \\
\hline $\mathrm{O} 3$ & $0.0184(9)$ & $0.0185(9)$ & $0.0134(7)$ & $-0.0014(7)$ & $0.0072(7)$ & $-0.0006(6)$ \\
\hline $\mathrm{O} 4$ & $0.0131(9)$ & $0.0184(9)$ & $0.0280(9)$ & $-0.0007(7)$ & $0.0097(7)$ & $-0.0012(7)$ \\
\hline O5 & $0.0130(8)$ & $0.0177(9)$ & $0.0135(7)$ & $0.0023(7)$ & $0.0052(6)$ & $0.0018(6)$ \\
\hline O6 & $0.0195(10)$ & $0.0120(8)$ & $0.0246(8)$ & $-0.0022(7)$ & $0.0067(7)$ & $0.0015(7)$ \\
\hline
\end{tabular}


Table S3. Angles in $\left(^{\circ}\right)$ for $\mathrm{Ag}_{2-x} \mathrm{Na}_{x} \mathrm{Mn}_{2} \mathrm{Fe}\left(\mathrm{VO}_{4}\right)_{3}(x=0,5 / 4$, and 2). Average angles are given in brackets.

\begin{tabular}{llll}
\hline & $\begin{array}{l}\mathbf{N a}_{2} \mathrm{Mn}_{2} \mathrm{Fe}\left(\mathrm{VO}_{4}\right)_{3} \\
\text { Angles }\end{array}$ & $\begin{array}{l}\mathrm{Ag}_{3 / 4} \mathrm{Na}_{5 / 4} \mathrm{Mn}_{2} \mathrm{Fe}\left(\mathrm{VO}_{4}\right)_{3} \\
\text { Angles }\end{array}$ & $\begin{array}{l}\mathrm{Ag}_{2} \mathrm{Mn}_{2} \mathrm{Fe}\left(\mathrm{VO}_{4}\right)_{3} \\
\text { Angles }\end{array}$ \\
\hline O6-V1-O6 & $100.99(13)$ & $102.12(12)$ & $103.19(10)$ \\
O5-V1-O6 $(\times 2)$ & $108.21(12)$ & $108.26(12)$ & $108.36(10)$ \\
O5-V1-O6 $(\times 2)$ & $111.63(10)$ & $111.07(10)$ & $110.74(8)$ \\
O5-V1-O5 & $115.27(12)$ & $115.28(10)$ & $114.82(9)$ \\
& $<109.32>$ & $<109.34>$ & $<109.37>$ \\
O1-V2-O2 & $106.13(11)$ & $106.76(10)$ & $107.18(9)$ \\
O3-V2-O4 & $106.61(11)$ & $106.52(11)$ & $106.70(9)$ \\
O1-V2-O3 & $110.48(13)$ & $110.69(13)$ & $110.82(12)$ \\
O2-V2-O4 & $110.57(13)$ & $109.76(12)$ & $109.01(10)$ \\
O1-V2-O4 & $111.29(13)$ & $111.25(12)$ & $111.43(11)$ \\
O2-V2-O3 & $111.83(11)$ & $111.92(10)$ & $111.73(9)$ \\
& $<109.48>$ & $<109.48>$ & $<109.48>$ \\
\hline
\end{tabular}



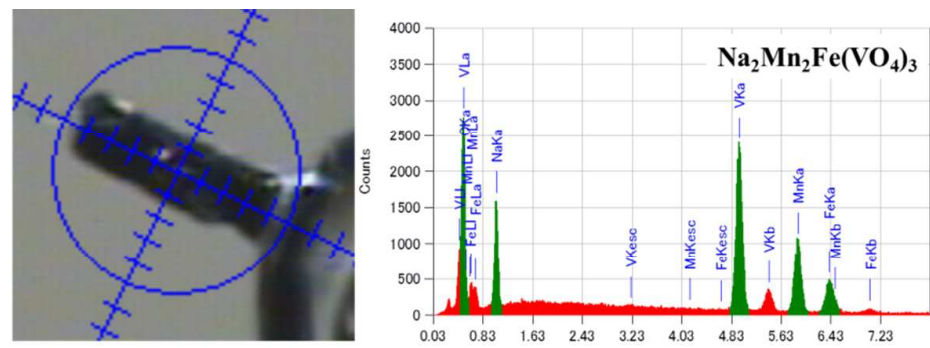

\begin{tabular}{|l|l|l|}
\hline Element & $\begin{array}{l}\text { Mass } \\
\%\end{array}$ & $\begin{array}{l}\text { Atom } \\
\%\end{array}$ \\
\hline Na K & 9.52 & 15.90 \\
\hline Mn K & 27.47 & 19.19 \\
\hline Fe K & 14.01 & 9.63 \\
\hline V K & 37.86 & 28.53 \\
\hline O K & 11.15 & 26.76 \\
\hline Total & 100.00 & 100.00 \\
\hline
\end{tabular}
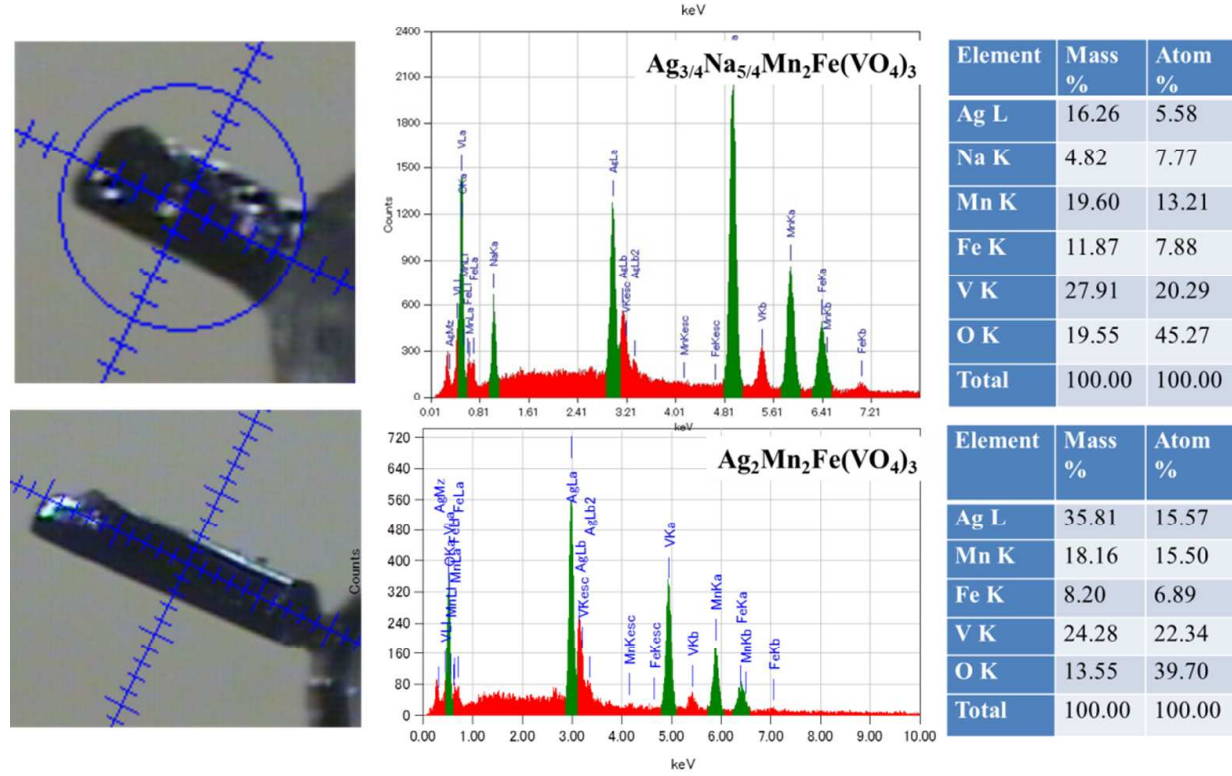

\begin{tabular}{|l|l|l|}
\hline & $\%$ & $\%$ \\
\hline Ag L & 16.26 & 5.58 \\
\hline Na K & 4.82 & 7.77 \\
\hline Mn K & 19.60 & 13.21 \\
\hline Fe K & 11.87 & 7.88 \\
\hline V K & 27.91 & 20.29 \\
\hline O K & 19.55 & 45.27 \\
\hline Total & 100.00 & 100.00 \\
\hline
\end{tabular}

\begin{tabular}{|l|l|l|}
\hline Element & $\begin{array}{l}\text { Mass } \\
\%\end{array}$ & $\begin{array}{l}\text { Atom } \\
\%\end{array}$ \\
\hline Ag L & 35.81 & 15.57 \\
\hline Mn K & 18.16 & 15.50 \\
\hline Fe K & 8.20 & 6.89 \\
\hline V K & 24.28 & 22.34 \\
\hline O K & 13.55 & 39.70 \\
\hline Total & 100.00 & 100.00 \\
\hline & &
\end{tabular}

Figure S1. EDX analyses of the single crystals used for the data collection. 


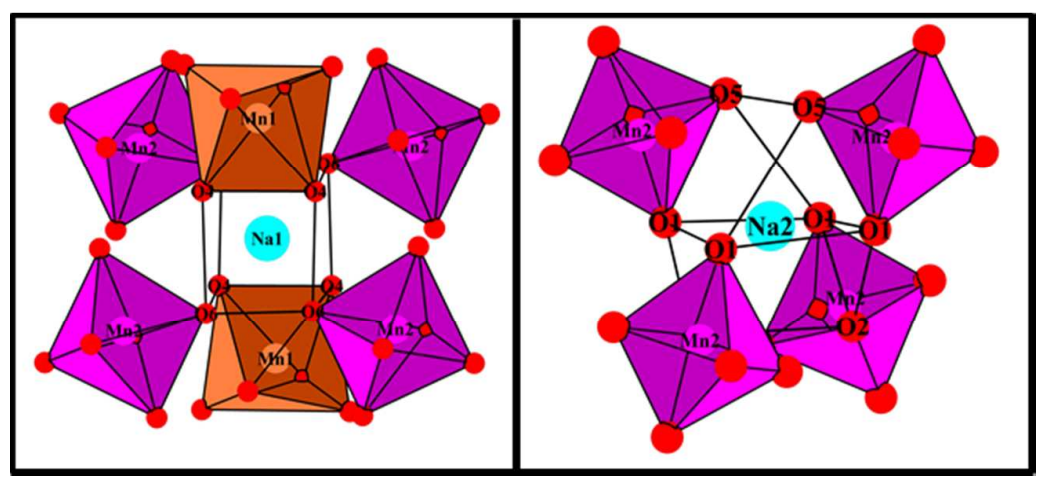

Figure S2. Coordination spheres of the $\mathrm{Na} 1$ and $\mathrm{Na} 2$ cations in $\mathrm{Na}_{2} \mathrm{Mn}_{2} \mathrm{Fe}\left(\mathrm{VO}_{4}\right)_{3}$. 

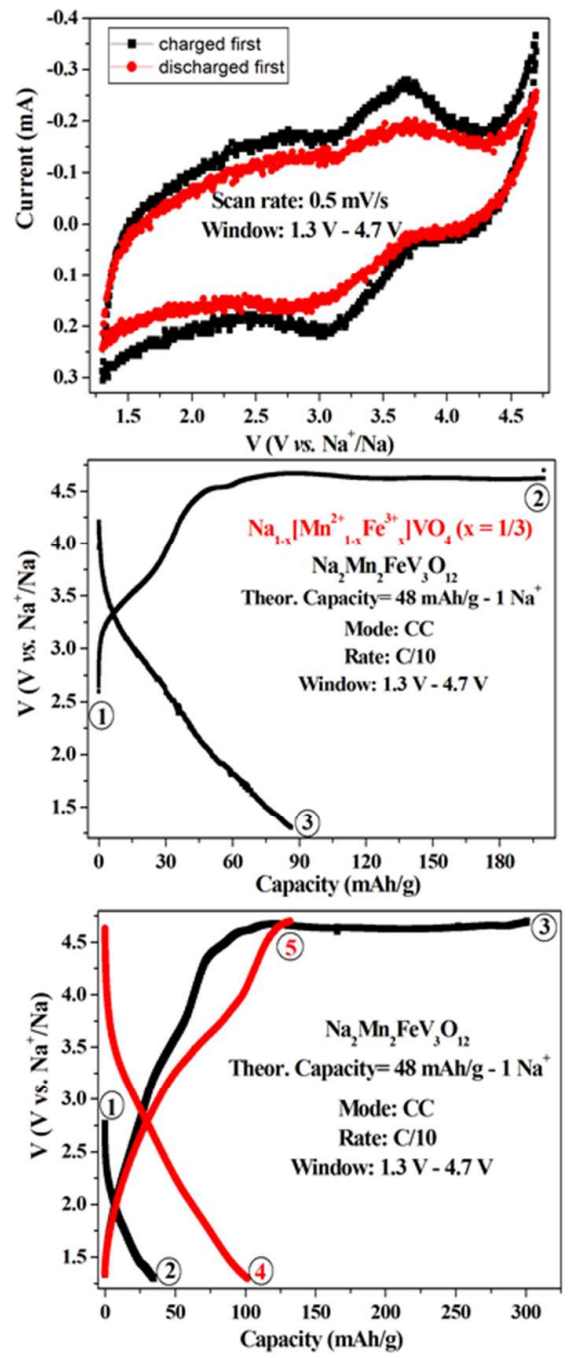

Figure S3. Charge/Discharge and cyclic voltammogram $(\mathrm{CV})$ curves of $\mathrm{Na}_{2} \mathrm{Mn}_{2} \mathrm{Fe}\left(\mathrm{VO}_{4}\right)_{3}$ recorded at room temperature, between 1.3 and $4.7 \mathrm{~V} v s . \mathrm{Na}^{+} / \mathrm{Na}$. The first cell has been charged first (middle) and the second cell has been discharged first (bottom). 


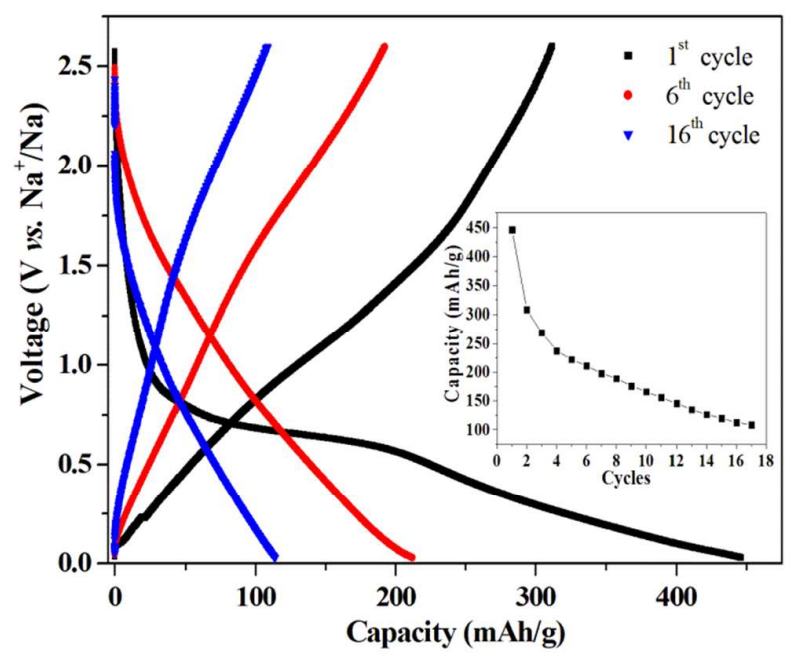

Figure S4. Charge/Discharge curves of $\mathrm{Na}_{2} \mathrm{Mn}_{2} \mathrm{Fe}\left(\mathrm{VO}_{4}\right)_{3}$ recorded at room temperature, between $\mathrm{V}$ and $0.03 \mathrm{~V} v s . \mathrm{Na}^{+} / \mathrm{Na}$ at a rate of $\mathrm{C} / 10$. The inset displays the capacity retention after seventeen cycles. 